\title{
Meromorphic extension of the zeta function for Axiom A flows
}

\author{
NICOLAI T. A. HAYDN \\ Department of Mathematics, University of Toronto, Toronto M5S 1A1, Canada
}

(Received 22 April 1988, revised 10 October 1988)

\begin{abstract}
We prove the meromorphicity of the zeta function on shifts of finite type for Hölder continuous functions assuming that the essential spectrum of the associated Ruelle operator is contained in the open unit disc. This result allows to extend the region of meromorphicity of the zeta function for Axiom A flows by a strip whose width is determined by the contraction rate of the flow.
\end{abstract}

\section{Introduction}

The classification of dynamical systems consists mainly in the search for invariants one of which (to some degree) is the zeta function. In general the zeta function is not very accessible, Axiom A systems being an exception. In this case the most interesting applications are to determine exponential decay of correlation functions (see for instance [12]) and to prove that closed orbits are equidistributed. Concerning the latter, the foundation to a number of recent results, most of which involve Ikehara's Tauberian theorem, was laid in [8]. The introduction of Markov partitions makes it much easier to extract properties of Axiom A diffeomorphisms. However these partitions are not canonical and it is a constant problem whether a result obtained for a Markov chain also applies to the underlying dynamical system. By a result of Manning [6] it is known that the zeta function of an Axiom A diffeomorphism is rational given by zeta functions on certain subshifts (which are rational themselves). According to Bowen [3] a similar result concerning meromorphicity applies to flows.

The main result of this paper, Theorem 4, is proved for the Banach space $C_{u}(\Sigma)$ of exponentially decreasing functions (with rate $e^{-u}<1$ ) on shifts of finite type. Usually as in [2, 9-12] $u$ is taken to be a constant, here however we embark upon a generalization and shall introduce modulus of continuity as we call $\boldsymbol{u}$ which will be a continuous and strictly positive function on the subshift. For piecewise monotone transformations of the unit interval and locally constant functions, Hofbauer and Keller [5] proved meromorphicity of the zeta function in the case where the essential spectrum of the transfer operator has radius less than 1 . In this case the spectrum consists of countably many discrete eigenvalues of finite multiplicity and an essential spectrum which is a disc centered at 0 . In analogy to interval 
maps, Pollicott [10] Theorem 1, proved that under appropriate conditions the spectrum of the Ruelle operator acting on $C_{\theta}(\Sigma)$ looks the same for constant $\theta>0$. In Lemma 2 we shall give a bound on the essential spectrum of the Ruelle operator on the Banach space of functions which decrease at a rate determined by a continuous function $u$ which is the modulus of continuity.

The aim of the present note is to extend further results previously obtained by Ruelle [11], Parry [9] and Pollicott [10] and to establish that the zeta function can be meromorphically continued as long as the essential spectrum of Ruelle's operator is contained in the open unit disc. This result also allows to extend the region of meromorphicity of the zeta function for Axiom A flows by half the contraction parameter beyond the halfplane in which it is holomorphic.

\section{Two sided subshift of finite type}

Let $A$ be some finite set with the discrete topology, let $A$ be an $|A| \times|A|$-matrix of ones and zeros and define

$$
\Sigma=\left\{z \in \prod_{i \in \mathbb{Z}} \mathbb{A}: A\left[z_{i}, z_{i+1}\right]=1 \forall i \in \mathbb{Z}\right\},
$$

which we give the product topology. We call $\Sigma$ a subshift of finite type and $A$ its alphabet. Define on $\Sigma$ the two-sided shift transformation by $\sigma(z)_{i}=z_{i+1}, i \in \mathbb{Z}$. The shiftspace $\Sigma$ has a natural set of Hölder equivalent metrics given by $d(x, y)=e^{-\alpha k(x, y)}$, where $k(x, y)$ is largest $k$ such that $x_{i}=y_{i},|i| \leq k$, and $\alpha$ is a positive number. A basis for the topology on $\Sigma$ is given by the open closed sets

$$
U\left(x_{-n} \cdots x_{n}\right)=\left\{z \in \Sigma: z_{i}=x_{i},|i| \leq n\right\},
$$

$n=1, \ldots$, where $x_{-n} \cdots x_{n}$ are words in $\Sigma$ of length $2 n+1$. Usually $U\left(x_{-n} \cdots x_{n}\right)$ is called a cylinder. Note that $\Sigma$ has dimension zero. The variation of a complex function $f$ on $\Sigma$ is defined by

$$
\operatorname{var}_{n} f(x)=\sup \{|f(x)-f(y)|: y \in \Sigma \text { satisfying } k(x, y) \geq n\},
$$

$n=1,2, \ldots$, and is a real and positive function on $\Sigma$. Let $u: \Sigma \rightarrow \mathbb{R}$ be strictly positive and continuous, then if the variation decays fast enough as $\boldsymbol{n}$ goes to infinity we have

$$
\operatorname{var}_{n} f(x) \leq c \cdot \exp \left(-2 \cdot \min \left(u^{n}(x), u^{-n}(x)\right)\right),
$$

$x \in \Sigma$, for some constant $c$. Here we used the abbreviation $u^{n}(x)$ for the sum $u(x)+u \sigma(x)+\cdots+u \sigma^{n}(x)$ and $u^{-n}(x)$ for $u \sigma^{-1}(x)+\cdots u \sigma^{-n}(x)$. If $\|f\|_{u}$ is the smallest possible constant $c$ which satisfies the above inequality, then $\|\cdot\| \|_{u}=$ $\|\cdot\|_{\infty}+\|\cdot\|_{u}$ is a norm, and the set of complex functions on $\Sigma$ which are finite with respect to this norm is a Banach algebra which we denote by $C_{u}(\Sigma)$. We have a filtration $C_{u}(\Sigma) \subset C_{u^{\prime}}(\Sigma), 0<u^{\prime} \leq u$, where $C_{u}(\Sigma)$ is dense in $C_{u^{\prime}}(\Sigma)$, with $\bigcap_{u>0} C_{u}(\Sigma)$ dense in $C_{u^{\prime}}(\Sigma)$. We call $u$ modulus of continuity. For convenience we shall assume that the variation of $u$ itself decays exponentially fast. If the modulus of continuity is a constant $\theta>0$, we get the usual Banach spaces of uniformly exponentially decreasing functions in which case $\|f\|_{\theta}$ is the best possible Hölder constant of $f$ 
(or Lipshitz constant if the metric defined uses $\theta$ in the place of $\alpha$ ). In $\S 5$ it will become clear that this setup is tailor-made for the analysis of Axiom A flows on manifolds. We shall assume that $(\Sigma, \sigma)$ is topologically mixing, in other words $A^{n}$ is positive for large enough $n$, where $A$ is the boolean matrix which defines the shift.

Let $\rho$ be a $\sigma$-invariant measure on $\Sigma$ and denote by $h(\rho)$ its measure theoretic entropy. For continuous $f: \Sigma \rightarrow \mathbb{R}$ the pressure is defined by

$$
P(f)=\sup _{\rho}\left(h(\rho)+\int f d \rho\right),
$$

where the supremum is taken over all $\sigma$-invariant probability measures on $\Sigma$. If $f$ is a real valued function in $C_{u}(\Sigma)$, some $u>0$, the supremum is always attained by a unique invariant measure called the equilibrium state for $f$. From [2] we know that the pressure equals

$$
\lim _{m \rightarrow \infty} m^{-1} \log \sum_{x \in \mathbf{F}(m)} \exp f^{m}(x)
$$

where $F(m)=\left\{x \in \Sigma: \sigma^{m} x=x\right\}$ denotes the periodic points of period $m$. The pressure function $P(f)$ is Lipshitz continuous in the supremum norm (with Lipshitz constant 1) on the real functions of $C_{u}(\Sigma)$ and has an analytic continuation to an open neighbourhood containing the real valued functions (cf. [9]). The topological entropy of $\sigma, h(\sigma)=\sup _{\rho} h(\rho)$, is equal to $P(0)$.

\section{The one-sided case}

Some questions, one of which is the meromorphicity of the zeta function, are more easily studied for one-sided shifts. For that purpose let us introduce the one-sided shiftspace

$$
\Sigma_{0}=\left\{y \in \prod_{i \geq 1} A: A\left[y_{i}, y_{i+1}\right]=1 \forall i \geq 1\right\} .
$$

The shift $\sigma: \Sigma_{0} \rightarrow \Sigma_{0}$ induced by the two-sided shift on $\Sigma$ is locally a homeomorphism and finite (at most $|\mathbb{A}|$ ) to one. Let $u_{0}$ be a strictly positive and continuous one-sided function. For $f_{0}: \Sigma_{0} \rightarrow \mathbb{C}$ the variation is given by $(k(x, y)$ as in the two-sided case)

$$
\operatorname{var}_{t} f_{0}(x)=\sup \left\{\left|f_{0}(x)-f_{0}(y)\right|: k(x, y) \geq t\right\},
$$

$t \geq 1$, and a norm is defined by $\left\|f_{0}\right\|_{u_{0}}=\left\|f_{0}\right\|_{\infty}+\left\|f_{0}\right\|_{u_{0}}$, where the Hölder constant $\left\|f_{0}\right\|_{u_{0}}$ which here is equal to $\sup _{x \in \Sigma} \sup _{t \geq 1} \exp \left(-u_{0}^{t}(x)\right) \operatorname{var}_{t} f_{0}(x)$ exists if the variation decays fast enough. As in the two-sided case we put $C_{u}\left(\Sigma_{0}\right)$ for the Banach space of all functions which are finite in the $\|\cdot \cdot\|_{u_{0}}$-norm and call $u_{0}$ modulus of continuity.

Two functions $f, g \in C_{u^{\prime}}(\Sigma)$ are said to be cohomologous if there exists a $v \in C_{u^{\prime}}(\Sigma)$ for some positive modulus of continuity $u^{\prime}$ such that $f-g=v-v \sigma$. A function that is cohomologous to zero is a cocycle. The zeta function for some $f \in C_{u}(\Sigma)$ is invariant under adding cocycles which leaves some freedom for the choice of $f$. An important and useful result involving the manipulation of Hölder continuous functions by cocyles is the following result by Sinai [13] of which we will give a formulation suitable to our context. 
Proposition 1. For $f \in C_{u}(\Sigma)$ there exists $v_{0}, f_{0} \in C_{\frac{1}{2} u}(\Sigma)$ such that $f_{0}=f+v_{0}-v_{0} \sigma$ satisfies $f_{0}(x)=f_{0}(y)$ whenever $x_{i}=y_{i}, i \leq 0$.

Proof. We adapt the proof given in [2, p. 11] for constant modulus of continuity $(u=\theta \cdot \tau, \theta>0)$ to our purpose. For each symbol $a \in \mathbb{A}$ pick an allowed left infinite sequence $\hat{y}=\cdots \hat{y}_{-1} \hat{y}_{0}$ such that $A\left[\hat{y}_{0}, a\right]=1$ and define a map $\pi: \Sigma \rightarrow \Sigma$ by $\pi(x)=$ $\cdots \hat{y}_{-1} \hat{y}_{0} x_{1} x_{2} \cdots, x \in \Sigma$, with $\hat{y}$ depending merely on $x_{1}$. We define $v_{0}=$ $\sum_{i \geq 0}\left(f \sigma^{i} \pi-f \sigma^{i}\right)$ and put

$$
f_{0}=f+v_{0}-v_{0} \sigma=f \pi+\sum_{i \geq 0}\left(f \sigma^{i+1} \pi-f \sigma^{i} \pi \sigma\right),
$$

which depends only on positive coordinates. It is clear that the summation over $i$ converges absolutely. We have to prove that $v_{0}$ and $f_{0}$ are as claimed in the Banach space $C_{\frac{1}{2} u}(\Sigma)$. We show this for $f_{0}$ and leave it to the reader to figure out that $v_{0} \in C_{\frac{1}{2} u}(\Sigma)$. To estimate the variation of $f_{0}$ we have to consider

$$
\begin{aligned}
f_{0}(x)-f_{0}(y)= & \sum_{i \geq 0} F 4\left(f \sigma^{i} \pi x-f \sigma^{i} \pi y+f \sigma^{i} \pi \sigma y-f \sigma^{i} \pi \sigma x\right) \\
= & \sum_{0 \leq i<j}\left(f \sigma^{i} \pi x-f \sigma^{i} \pi y+f \sigma^{i} \pi \sigma y-f \sigma^{i} \pi \sigma x\right) \\
& +\sum_{i \geq j}\left(f \sigma^{i+1} \pi y-f \sigma^{i} \pi \sigma y+f \sigma^{i+1} \pi x-f \sigma^{i} \pi \sigma x\right),
\end{aligned}
$$

where $x, y$ are points in $\Sigma$ such that $x_{i}=y_{i},|i| \leq k$, for some positive $k$. With $j \in(0, k)$ an integer chosen such that $\left|u^{k-j}(x)-u^{-j}(x)\right| \leq\|u\|_{\infty}$ we get

$$
\begin{aligned}
\left|f_{0}(x)-f_{0}(y)\right| & \leqq c_{1} \sum_{0 \leq i<j} \exp -2 u^{k-i}\left(\sigma^{i} \pi x\right)+c_{2} \sum_{i \geq j} \exp -2 u^{i}\left(\sigma^{i} \pi x\right) \\
& \leq c_{3} \exp -\left(u^{k-j}\left(\sigma^{j} \pi x\right)+u^{j}\left(\sigma^{j} \pi x\right)\right) \\
& \leq c_{3} \exp -u^{k}(\pi x)
\end{aligned}
$$

for some $c_{1}$ since inf $u>0$ and $c_{2}, c_{3}>0$. Thus $\left(c_{4}>0\right)$

$$
\operatorname{var}_{k} f_{0}(x) \leq c_{4} \exp -u_{0}^{k}(x)
$$

where $u_{0}=u \pi+\sum_{i \geq 0}\left(u \sigma^{i+1} \pi-u \sigma^{i} \pi \sigma\right)$ depends only on positive coordinates and is cohomologous to $u$. Note that $u_{0}$ is well-defined since we assumed that $u$ has exponentially fast decaying variation. Thus $f_{0}$ can be identified with an element in $C_{u_{0}}\left(\Sigma_{0}\right)$ where $u_{0}$ is a one-sided modulus of continuity. A priori we do no know whether $u_{0}$ is strictly positive, however as $u_{0}$ is cohomologous to $u$ one easily sees there exists an integer $n$ and a constant $c_{5}>0$ such that $u_{0}^{k} \geq c_{5}(k-n)$ for $k \geq n$. Nevertheless in the following we shall for simplicity assume that $u_{0}$ is strictly positive.

From now on we drop the index 0 and without fear of confusion we denote the one-sided shift by $\Sigma$. Given a (one-sided) Hölder continuous function $f \in C_{u}(\Sigma)$, then, following Ruelle, one defines a Perron-Frobenius operator (transfer matrix) $L: C_{u}(\Sigma) \rightarrow C_{u}(\Sigma)$ by setting:

$$
(L \chi)(x)=\sum_{x^{\prime} \in \sigma^{-1} x} \chi\left(x^{\prime}\right) \exp f\left(x^{\prime}\right), \quad \chi \in C_{u}(\Sigma),
$$


where the summation is over all $x^{\prime} \in \Sigma$ satisfying $\sigma x^{\prime}=x$. Let $T_{n}, n \geq 1$, be the set of all $\Sigma$-words of length $n$ and set $U(\eta), \eta \in T_{n}$, for the cylinder $\left\{y \in \Sigma: \eta_{i}=y_{i}\right.$, $i=1, \ldots, n\}$. Put $f^{n}(y)$ for the sum $f(y)+\cdots+f \sigma^{n-1}(y)$ and one easily verifies

$$
\left(L^{n} \chi\right)(x)=\sum_{\eta} \chi(\eta x) \exp f^{n}(\eta x)
$$

where the summation is over all $\eta \in T_{n}$, such that the concatenation $\eta x$ is an admissible sequence in $\Sigma$. As a consequence of the Perron-Frobenius theorem for real valued $f$ the largest eigenvalue $\lambda_{0}$ of the associated operator $L_{f}$ is simple and real while the rest of the spectrum is contained in a disc of radius strictly smaller than $\lambda_{0}$ (see [11, Proposition 5.24]). Moreover $\log \lambda_{0}$ is the pressure of $f$ as defined by the variational principle. For complex $f$ we can now state the main result of this section.

LEMMA 2. The essential spectrum of $L_{f}$ has radius $\leq e^{P(\mathbb{R f}-u)}$.

Proof. For $u$ constant $\theta>0$ the lemma reduces to the result given in [10, Theorem 1]. We adapt the proof given there suitably. It is known that the measure $\omega$ of maximal entropy is positive on open sets and non atomic ( $\omega$ is the Parry measure, see [14, Theorem 8.10]). Fix $n \geq 1$ and denote by $\omega_{\eta}, \eta \in T_{n}$, the induced measure on $U(\eta)$, that is $\omega_{\eta}(\chi)=\omega\left(\chi_{\eta} \chi\right) / \omega\left(\chi_{\eta}\right)$ for (test) functions $x \in C_{u}(\Sigma)$. The radius of the essential spectrum is by Nussbaum's formula [7] the limit of (inf $\left.\left\|L_{f}^{n}-K_{n}\right\| \|_{u}\right)^{1 / n}$ for $n \rightarrow \infty$, where the infimum is over compact operators $K_{n}$ on $C_{u}(\Sigma)$. For $K_{n}$ we take $L_{f}^{n}$ restricted to locally constant functions. We put $K_{n}=L_{f}^{n} \circ S_{n}$, where $S_{n}$ maps $C_{u}(\Sigma)$ onto functions which are constant on cylinders of the form $U(\eta), \eta \in T_{n}$, and is given by

$$
\left(S_{n} g\right)=\sum_{\eta} \omega_{\eta}(g) \chi_{\eta}
$$

$g \in C_{u}(\Sigma)$. Pick some $g \in C_{u}(\Sigma)$ and put $\chi=\left(\right.$ id $\left.-S_{n}\right) g$ for which one immediately deduces the estimate $|\chi(\eta x)| \leq\|g\|_{u} \exp -u^{n}(\eta x), x \in V(\eta)=\{z \in \Sigma: \eta z \in \Sigma\}, \eta \in T_{n}$. By the variational principle we obtain $\left(c_{6}>0\right)$

$$
\left|\left(L^{n} \chi\right)(x)\right| \leq \sum_{\eta}|\chi(\eta x)| \cdot\left|\exp f^{n}(\eta x)\right| \leq c_{6} e^{n P(\boldsymbol{R} f-u)}
$$

and therefore $\left\|\left(L^{n} \chi\right)\right\|_{\infty} \leq c_{6} e^{n P(R f-u)}$ for $n=1,2, \ldots$ To estimate the variation of $L^{n} \chi$ we need bounds on the variation of $\chi$ as

$$
\operatorname{var}_{k} \chi(x) \leq \begin{cases}2 \cdot \max \left\{\operatorname{var}_{n} g(y): k(x, y)=k\right\} & \text { for } k \leq n, \\ \operatorname{var}_{k} g(x) & \text { for } k>n .\end{cases}
$$

Now let $x, y$ be two points in $\Sigma$ which share the same symbols on the coordinates form 1 to $k$. Thus $\left(c_{7}, c_{8}>0\right)$

$$
\begin{aligned}
&\left|\left(L^{n} \chi\right)(x)-\left(L^{n} \chi\right)(y)\right| \\
& \leq \sum_{\eta}|\chi(\eta x)-\chi(\eta y)| \exp \mathbb{R} f^{n}(\eta x)+\sum_{\eta}|\chi(\eta y)| \cdot 1 \\
& \quad-\exp \left(f^{n}(\eta y)-f^{n}(\eta x)\right) \exp \mathbb{R} f^{n}(\eta x) \\
& \leq \sum_{\eta}\|g\|_{u} \exp \left(\mathbb{R} f^{n}(\eta x)-u^{n+k}(\eta x)\right)+c_{7} \sum_{\eta}|\chi(\eta y)| \exp \left(\mathbb{R} f^{n}(\eta x)-u^{k}(x)\right) \\
& \leq c_{8}\|g\|_{u} e^{n P(\mathbf{R} f-u)} \exp -u^{k}(x)
\end{aligned}
$$


and for the Hölder constant we get $\left\|\left(L^{n} \chi\right)\right\|_{u} \leq c_{8}\|g\|_{u} e^{n P(\mathbf{R} f-u)}, n=1,2, \ldots$ Hence the lemma.

It is clear that $L=L_{f}$ has discrete eigenvalues of finite multiplicity in the annulus $\left\{z \in \mathbb{C}: e^{P(\mathbf{R} f-u)}<|z| \leq e^{P(\mathbf{R} f)}\right\}$ see $[\mathbf{1 0}]$. Let $\mathbb{E}_{\lambda}$ be the eigenspace in $C_{u}(\Sigma)$ associated to the eigenvalue $\lambda,|\lambda|>e^{P(R f-u)}$, and $\mathbb{E}_{\lambda}^{*}$ the corresponding eigenspace in the dual $C_{u}(\Sigma)^{*}$. We have $\operatorname{dim} \mathbb{E}_{\lambda}=\operatorname{dim} \mathbb{E}_{\lambda}^{*}$ as complex vectorspaces. For real $f$ the eigenspaces corresponding to the largest eigenvalue are one dimensional and spanned by $\mu$ and $M$ respectively. (The equilibrium state for $f$ is given by $M \mu$, see [2].) In $\mathbb{E}_{\lambda}^{*}, \mathbb{E}_{\lambda}$ we choose an orthogonal basis $\mu_{\lambda, r}, M_{\lambda, r}, r=1, \ldots, \operatorname{dim} \mathbb{E}_{\lambda}$, normalized so that $\mu_{\lambda, r}\left(M_{\lambda^{\prime}, s}\right)=\delta_{\lambda, \lambda} \delta_{r, s}$, where $\delta_{r, s}$ is the Kronecker symbol: $\delta_{r, s}=1$ if $r=s$ and 0 otherwise. The projections $\mathbb{P}_{\lambda}$ from $C_{u}(\Sigma)$ onto the eigenspaces $\mathbb{E}_{\lambda}$ then take the form of a scalar product $M_{\lambda}^{\perp} \mu_{\lambda}(\cdot)$, where $M_{\lambda}$ is the vector of eigenfunctions $\left(M_{\lambda, 1}, M_{\lambda, 2}, \ldots\right)$ and similarly $\mu_{\lambda}$ is the vector of functionals $\left(\mu_{\lambda, 1}, \mu_{\lambda, 2}, \ldots\right)\left({ }^{1}\right.$ denotes transposition). We can decompose the transfer operator as follows

$$
L(\chi)=\sum_{\lambda} \lambda M_{\lambda}^{\perp} L_{\lambda} \mu_{\lambda}(\chi)+R(\chi),
$$

where the sum is over all discrete eigenvalues $\lambda$ whose modulus is larger than $e^{P(R f-u)}$. The remainder $R=L \mathbb{P}: C_{u}(\Sigma) \rightarrow C_{u}(\Sigma)$ has spectral radius $\leq e^{P(R f-u)}$, where $\mathbb{P}=\mathrm{id}-\sum_{\lambda} \mathbb{P}_{\lambda}$ (summation again over the discrete spectrum) is the projection onto the subspace of $C_{u}(\Sigma)$ orthogonal to the eigenspaces $\mathbb{E}_{\lambda},|\lambda|>e^{P(R f-u)}$. We assume the matrices $L_{\lambda}$ are in Jordan normal form (with ones in the diagonal).

\section{The Zeta function}

Let $\Sigma$ be the one-sided subshift associated with the transition matrix $A$ and define for $f \in C_{u}(\Sigma)$ the zeta function

$$
\zeta(f)=\exp \sum_{m \in \mathbb{N}} \zeta_{m} / m
$$

where the summation in $\zeta_{m}(f)=\sum_{x \in F(m)} \exp f^{m}(x)$ is over periodic points $x$ of period $m$. It is clear that $\zeta(f)$ is non-zero and analytic whenever the summation converges. Furthermore, the slightly more general zeta function

$$
d(z, f)=\zeta(f+\log z)=\exp \sum_{m \in \mathbb{N}} z^{m} \zeta_{m} / m,
$$

is non-zero and analytic for $|z|<e^{-P(\mathbf{R} f)}$. The first substantial step in extending the region of analyticity was achieved by Ruelle for constant modulus of continuity $\theta>0$.

THEOREM 3. ([11, Theorem 5.29]). For real $f$ there exists a function $R(f)>-P(f)$ such that $d(z, f)$ is analytic for $|z|<e^{R(f)}$ with the exception of a single pole at $e^{-P(f)}$.

This theorem contains already the ingredients for the further improvements made subsequently. The proof essentially makes use of the fact that the largest eigenvalue $e^{P(f)}$ of the $L_{f}$ operator is real and single and the rest of the spectrum is contained in a disc of radius strictly less than $e^{P(f)}$. The function $f$ is approximated by stepfunctions $f_{m}$ which are constant on cylinders of the form $U\left(x_{1} \cdots x_{n}\right)$ where the length of the words $x_{1} \cdots x_{n}$ depends linearly on $m$, that is $n=\alpha m$. $0<\alpha<1$. 
With perturbation theory on Banach spaces it then follows that $d(z, f)$ has the claimed meromorphic extension. This result was generalized by Parry, [9, Theorem $1]$, to a neighbourhood of the real valued functions in the complex space $C_{\theta}(\Sigma)$. Using a more complete characterization of the spectrum of the transfer operator, Pollicott [10, Theorem 2], showed that the proof of Ruelle's theorem can in fact be adapted to the situation where one has to single out a larger number of eigenvalues, thus replacing the function $R(f)$ by a constant $C(\theta)=\theta^{2} /(\theta+h)$ which depends only on the space $C_{\theta}(\Sigma), h$ being the topological entropy of the shift. However, since the only way to estimate the traces of the approximating transfer matrices modulo a subspace of some fixed dimension is by adding up the moduli of the eigenvalues whose number grows like $e^{n h}, C(\theta)$ depends explicitly on the topological entropy and yields particularly poor estimates if $h$ is large.

The aim of this paper is to establish meromorphicity of $d(z, f)$ in the disc $\left\{z \in \mathbb{C}:|z|<e^{-P(\boldsymbol{A} f-u)}\right\}$. We choose a more elementary approach which explicitly (however implicitly) does not involve perturbation theory but relies upon repeated use of the variational principle.

To show that the zeta function in general does not admit a meromorphic extension to the entire complex plane, Gallovotti [4] constructs a function $f$ such that $d(z, f)$ has an essential singularity at finite distance. By adapting this example suitably, Pollicott shows in $[10]$ that $d(z, f), f \in C_{\theta}(\Sigma)$, can have an essential singularity arbitrarily close to the disc of radius $e^{\theta-\boldsymbol{P}(\boldsymbol{R} f)}$. In view of this example, the result presented here is the best possible that can be obtained without further restriction other than on the pressure.

THEOREM 4. $\zeta(f)$ is analytic and non-zero for $\left\{f \in C_{u}(\Sigma): P(\mathbb{R} f)<0\right\}$ and extends in the function space meromorphically to the domain where $P(\mathbb{R} f-u)<0$.

For every $\delta \in A$ we choose $\hat{x} \in \Sigma$ such that $\delta \hat{x}$ is an element in $\Sigma$. We also write $\eta \hat{x} \in \Sigma, \eta \in T_{m}$, for the concatenation of $\eta$ with $\hat{x}=\hat{x}\left(\eta_{m}\right)$, define a complex function $F$ on $T_{m}$ by $F(\eta)=f^{m}(\eta \hat{x})$ and set $\Phi_{\eta}(x)=\exp \left(f^{m}(\eta x)-F(\eta)\right)$ whenever $x \in$ $V(\eta)=\{z \in \Sigma: \eta z \in \Sigma\}$ and 0 otherwise. It is clear that $\Phi_{\eta}(x)$ lies in $C_{u}(\Sigma)$. Denote by $\chi_{\eta}$ the characteristic function of $U(\eta)$. For the proof of Theorem 4 we shall need the following identity.

LeMmA 5: Let $\mu \in C_{u}(\Sigma)^{*}$ and $\eta \in T_{m}, m \geq 1$, then

$$
\left(L^{* m} \mu\right)\left(e^{-F(\eta)} \chi_{\eta}\right)=\mu\left(\Phi_{\eta}\right) \text {. }
$$

Proof. We do the manipulations

$$
\begin{aligned}
\left(L^{* m} \mu\right)\left(e^{-F(\eta)} \chi_{\eta}\right) & =\int_{V(\eta)} e^{-F(\eta)}\left(L^{m} \chi_{\eta}\right)(x) d \mu(x) \\
& =\int_{V(\eta)} \sum_{v} \chi_{\eta}(v x) \exp \left(f^{n}(v x)-F(\eta)\right) d \mu(x)=\mu\left(\Phi_{\eta}\right),
\end{aligned}
$$

where the summation is over $v \in T_{m}$.

Proof of Theorem 4. The fact that $\zeta(f)$ is analytic and non-zero for $P(\mathbb{R} f)<0$ follows 
from the variational principle

$$
\underset{m \rightarrow \infty}{\lim \sup } \sqrt[m]{\zeta_{m}}(\mathbb{R} f)=e^{P(\mathbb{R} f)}<1 .
$$

The condition $P(\mathbb{R} f)<0$ implies that $L_{f}$ has spectral radius less than one. In the more general case we face here, one singles out a finite number of eigenvalues, in particular these with modulus $\geq 1$, and uses a similar argument applied to a remainder term which has spectral radius $\leq \rho<1$.

The proof splits into two parts. In the first step (I) we use the decomposition (1) of Ruelle's operator and show that $\zeta_{m}(f)-\sum_{|\lambda| \geq \rho} \lambda^{m}+R_{m}$ decays exponentially fast for $m \rightarrow \infty$, where the $\lambda$ 's are discrete eigenvalues of $L_{f}$. The remainder terms $R_{m}$ originate from the $R$-term in (1) plus the contribution made by discrete eigenvalues whose moduli are less than $\rho$. In the second part (II) we prove that the $R_{m}$ decay exponentially fast as $m$ goes to infiity and therefore add up to a function which is holomorphic in the halfplane given by $P(\mathbb{R} f-u)<0$.

Since $P=P(\mathbb{R} f-u)<0$ the essential spectrum of $L_{f}$ (and $L_{f}^{*}$ ) is contained in a disc with radius strictly less than one and all but a finite number of discrete eigenvalues are in modulus less than one. We shall show that $\zeta_{m}$ is approximately $\sum_{|\lambda| \geq \rho} \lambda^{m}$ where the remainder term decreases exponentially fast as $m$ goes to infinity.

(I) Obviously $\left(L^{m} \chi_{\eta}\right)(x)$ equals $\exp f^{m}(\eta x)$ for $x \in \Sigma$ such that the composition $\eta x$ is again in $\Sigma$ and zero otherwise. With $\omega_{\eta}, \eta \in T_{m}$, as in Lemma 2, we get that

$$
\omega_{\eta}\left(L^{m} \chi_{\eta}\right)=\omega\left(\chi_{\eta}\right)^{-1} \int_{U(\eta)} \exp f^{m}(\eta x) d \omega(x)
$$

is zero if $\eta \eta$ is not admissible in $\Sigma$ and otherwise satisfies the inequation

$$
\left|\omega_{\eta}\left(L^{m} \chi_{\eta}\right)-\exp f^{m}\left(\eta^{\infty}\right)\right| \leq c_{0}\left|\exp (f-u)^{m}\left(\eta^{\infty}\right)\right|,
$$

as $f$ has modulus of continuity $u$, for some $c_{0}<\infty$, where $\eta^{\infty}$ is the periodic point which one obtains by repeating the string $\eta$ infinitely often. Hence

$$
\left|\sum_{x \in \mathbf{F}(m)} \exp f^{m}(x)-\sum_{\eta} \omega_{\eta}\left(L^{m} \chi_{\eta}\right)\right| \leq c_{0} \zeta_{m}(\mathbb{R} f-u) \leq c_{1} e^{m P} \leq c_{1} \rho^{m},
$$

where the summation is over $\eta \in T_{m}$. Here we made use of the part of the variational principle which implies that $\zeta_{m}(\mathbb{R} f-u) \leq$ const $\times e^{m P}$ (for details see Bowen [2, p. 32]). We want to show the second sum on the left hand side is up to some remainder terms equal to $\sum_{|\lambda| \geq \rho} \lambda^{m}$. For discrete eigenvalue $\lambda$ let $\mathbb{P}_{\lambda}$ be the projection onto the eigenspace $\mathbb{E}_{\lambda}$. Set $\mathbb{P}=\mathrm{id}-\sum_{|\lambda| \geq \rho} \mathbb{R}_{\lambda}$ and we obtain by (1)

$$
\sum_{\eta} \omega_{\eta}\left(L^{m} \chi_{\eta}\right)=\sum_{|\lambda| \geq \rho} \sum_{\eta} \omega_{\eta}\left(M_{\lambda}^{\perp}\right) \mu_{\lambda}\left(L^{m} \chi_{\eta}\right)+\sum_{\eta} \omega_{\eta}\left(L^{m} \mathbb{P} \chi_{\eta}\right) .
$$

Observe that the spectral radius of $L^{m} \mathbb{P}$ is bounded by $\rho^{m}$. Since there are only finitely many eigenfunctions $M_{\lambda}$ for which $|\lambda|$ is at least $\rho$ there exists a $c_{2}$ such that in the $C_{u}$-topology $\left\|M_{\lambda, r}\right\|_{u} \leq c_{2}$ and therefore

$$
\begin{gathered}
\left|\omega_{\eta}\left(M_{\lambda, r}\right)-M_{\lambda, r}(\eta x)\right| \leq c_{3} \exp -u^{m}(\eta x), x \in V(\eta), \quad \eta \in T_{m}, \\
r=1, \ldots, \operatorname{dim} \mathbb{E}_{\lambda}, \quad|\lambda| \geq \rho,
\end{gathered}
$$


with a new constant $c_{3}$. For $\hat{x}$ as in Lemma 5 depending on $\eta_{m}$ we find that the functions $S_{\lambda, r, \eta}(x)=\omega_{\eta}\left(M_{\lambda, r}\right) \nabla(x)-M_{\lambda, r}(\eta x)$ defined on $V(\eta)$ are in the supremum norm bounded by $c_{3} \exp -u^{m}(\eta \hat{x})$ and that their variations can be estimated as

$$
\operatorname{var}_{t} S_{\lambda, r, \eta}(x) \leq \operatorname{var}_{m+t} M_{\lambda, r}(\eta x) \text {. }
$$

Therefore $\left\|S_{\lambda, r, \eta}\right\|_{u} \leq c_{4} \exp -u^{m}(\eta \hat{x}), c_{4}>0$, and once more employing the variational principle we get

$$
\begin{aligned}
& \left|\sum_{\eta} \dot{\omega}_{\eta}\left(M_{\lambda, r}\right) \mu_{\lambda, s}\left(L^{m} \chi_{\eta}\right)-\lambda^{m}\left(L_{\lambda}^{m}\right)_{r, s}\right| \\
& \quad \leq \sum_{\eta}\left|\omega_{\eta}\left(M_{\lambda, r}\right) \mu_{\lambda, s}\left(L^{m} \chi_{\eta}\right)-\mu_{\lambda, s}\left(M_{\lambda, r}(\eta \cdot) L^{m} \chi_{\eta}\right)\right| \\
& \quad=\sum_{\eta}\left|\mu_{\lambda, s}\left(S_{\lambda, r, \eta} L^{m} \chi_{\eta}\right)\right| \\
& \quad \leq\left\|\left|\mu_{\lambda, s}\right|\right\|_{u} \sum_{\eta}\left\|S_{\lambda, r, \eta} L^{m} \chi_{\eta} \mid\right\|_{u} \\
& \quad \leq c_{5} \sum_{\eta}\left|\exp \left(-u^{m}(\eta \hat{x})+f^{m}(\eta \hat{x})\right)\right|,
\end{aligned}
$$

$c_{5}>0$, as the functionals $\mu_{\lambda, s},|\lambda| \geq \rho$, are in the $C_{u}^{*}$-norm uniformly bounded. Thus we see that for some $c_{6}$ involving the dimension of the largest eigenspace $\mathbb{E}_{\lambda}$

$$
\left|\sum_{\eta} \omega_{\eta}\left(M_{\lambda}^{\perp}\right) \mu_{\lambda}\left(L^{m} \chi_{\eta}\right)-\operatorname{trace}\left(\lambda L_{\lambda}\right)^{m}\right| \leq c_{6} e^{P m} \leq c_{6} \rho^{m}
$$

and $\left(c_{7}>0\right)$

$$
\left|\zeta_{m}-\sum_{|\lambda| \geq \rho} \lambda^{m}-\sum_{\eta} \omega_{\eta}\left(L^{m} \mathbb{P} \chi_{\eta}\right)\right| \leq c_{7} \rho^{m} .
$$

(II) We are finished if we can show that in the last inequality the third term on the left hand side goes exponentially fast to zero as $m$ goes to infinity. Lemma 5 applied to the functional $\mathbb{P}^{*} \omega_{\eta}$ yields

$$
\omega_{\eta}\left(L^{m} \mathbb{P} \chi_{\eta}\right)=e^{F(\eta)}\left(L^{* m} \mathbb{P}^{*} \omega_{\eta}\right)\left(e^{-F(\eta)} \chi_{\eta}\right)=e^{F(\eta)}\left(\mathbb{P}^{*} \omega_{\eta}\right)\left(\Phi_{\eta}\right) .
$$

We can replace $\left(\mathbb{P}^{*} \omega_{\eta}\right)\left(\Phi_{\eta}\right)$ by $\mathbb{P} \Phi_{\eta}$ evaluated at the point $\eta \hat{x}$ plus an error term. Hence

$$
\sum_{\eta} e^{F(\eta)} \omega_{\eta}\left(\mathbb{P} \Phi_{\eta}\right)=\sum_{\eta} e^{F(\eta)}\left(\left(\mathbb{P} \Phi_{\eta}\right)(\eta \hat{x})+R_{\eta}\right) .
$$

To estimate the remainder terms we observe that $\mathbb{P}$ is a bounded operator on $C_{u}(\Sigma)$ and $\Phi_{\eta}$ is bounded uniformly in $m$ and $\eta \in T_{m}$. We get

$$
\left|\omega_{\eta}\left(\mathbb{P} \Phi_{\eta}\right)-\left(\mathbb{P} \Phi_{\eta}\right)(\eta \hat{x})\right| \leq\left\|\mathbb{P} \Phi_{\eta}\right\|_{u} \exp -u^{m}(\eta \hat{x}) \leq c_{8} \exp -u^{m}(\eta \hat{x}),
$$

with some $c_{8}>0$, for all $\eta$. To estimate the contribution made by $R_{\eta}$, we sum over $\eta$ and find by the variational principle

$$
\sum_{\eta}\left|e^{F(\eta)}\right| \cdot\left|R_{\eta}\right| \leq c_{8} \sum_{\eta} \exp \left(\mathbb{R} F(\eta)-u^{m}(\eta \hat{x})\right) \leq c_{9} e^{m P} \leq c_{9} \rho^{m},
$$

$c_{9}>0$. To estimate the terms involving $\left(\mathbb{P} \Phi_{\eta}\right)(\eta \hat{x})$ it is necessary to decompose $\Phi_{\eta}$ as follows. For $k=1, \ldots, m$ note that the functions $\Psi_{k, \eta}(x)=$ $f \circ \sigma^{m-k}(\eta x)-f \circ \sigma^{m-k}(\eta \hat{x})$ are in the supremum norm bounded by 
$c_{10} \exp -u^{k}\left(\sigma^{m-k}(\eta x)\right)$ for some $c_{10} \leq\|f\|_{u} /(1-\exp (-\inf u))$. Moreover, since $\operatorname{var}_{t} \Psi_{k, \eta}(x) \leq \operatorname{var}_{t+k} f(x), x \in V(\eta)$, we see that $\left\|\Psi_{k, \eta}\right\|_{u}$ is for all $\eta$ bounded by $c_{10} \exp -u^{k}\left(\sigma^{m-k}(\eta \hat{x})\right)$. Now put $\Phi_{1, \eta}=\exp \Psi_{1, \eta}$ and define inductively

$$
\Phi_{i, \eta}=\Phi_{i-1, \eta}\left(\exp \Psi_{i, \eta}-1\right)=\exp \sum_{1 \leq k \leq i} \Psi_{k, \eta}-\exp \sum_{1 \leq k \leq i-1} \Psi_{k, \eta},
$$

$i=2, \ldots, m$. Clearly $\Phi_{\eta}=\sum_{1 \leq i \leq m} \Phi_{i, \eta}$ and as one easily verifies

$$
\left\|\Phi_{i, \eta}\right\|_{u} \leq c_{11} \exp -u^{i}\left(\sigma^{m-i}(\eta \hat{x})\right)
$$

$i=1, \ldots, m$, where $c_{11}$ depends on $c_{10}$ and the supremum norm of $f$. Note that $\Phi_{i, \eta}=\Phi_{i, \tilde{\eta}}$ whenever $\eta_{k}=\tilde{\eta}_{k}, k=1, \ldots, m-i+1$. We find

$$
\sum_{\eta} e^{F(\eta)}\left(\mathbb{P} \Phi_{\eta}\right)(\eta \hat{x})=\sum_{i} \sum_{\eta} e^{F(\eta)}\left(\mathbb{P} \Phi_{i, \eta}\right)(\eta \hat{x})=\sum_{i} \sum_{|\delta|=i} \sum_{v} e^{F(v \delta)}\left(\mathbb{P} \Phi_{i, v \delta}\right)(\eta \hat{x}),
$$

where the summation is over all $(v, \delta) \in T_{m-i} \times T_{i}$ such that $v \delta=\eta \in T_{m}$. To stress that $\Phi_{i, v \delta}$ is independent of $v$, we write $\varphi_{i, \delta}$ for $\Phi_{i, v \delta}$ and treat summation over $v, \delta$ and $i$ separately. For $i, \delta$ fixed we get

$$
\begin{aligned}
\left|\sum_{\nu} e^{F(v \delta)}\left(\mathbb{P} \Phi_{i, v \delta}\right)(v \delta \hat{x})\right| & =\left|\sum_{\nu}\left(\mathbb{P} \varphi_{i, \delta}\right)(v \delta \hat{x}) \exp f^{m-i}(v \delta \hat{x})\right| \cdot\left|\exp f^{i}(\delta \hat{x})\right| \\
& =\left|\left(L^{m-i} \mathbb{P} \varphi_{i, \delta}\right)(\delta \hat{x})\right| \exp \mathbb{R} f^{i}(\delta \hat{x}) \\
& \leq c_{12} \rho^{m-i}\left\|\varphi_{i, \delta}\right\|_{u} \exp \mathbb{R} f^{i}(\delta \hat{x}) \\
& \leq c_{12} c_{11} \rho^{m-i} \exp (\mathbb{R} f-u)^{i}(\delta \hat{x}),
\end{aligned}
$$

$c_{12}>0$. Here we made use of the fact that the spectral radius of $L^{m-i} \mathbb{P}$ is bounded by $\rho^{m-i}$. For the summation over $\delta$ and $i$ we borrow a last time from the variational principle and obtain

$$
\begin{aligned}
\left|\sum_{\eta} e^{F(\eta)} \omega_{\eta}\left(\mathbb{P} \Phi_{\eta}\right)\right| & \leq c_{9} \rho^{m}+\sum_{i} c_{12} c_{11} \rho^{m-i} \sum_{\delta} \exp (\mathbb{R} f-u)^{i}(\delta \hat{x}) \\
& \leq c_{9} \rho^{m}+\sum_{i} c_{12} c_{11} \rho^{m-i} c_{13} e^{i P},
\end{aligned}
$$

$c_{13}>0$. Hence the remainder which includes the contribution made by the essential spectrum of $L$ can be estimated as

$$
\left|\sum_{\eta} \omega_{\eta}\left(L^{m} \mathbb{P} \chi_{\eta}\right)\right| \leq\left(c_{9}+m c_{12} c_{11} c_{13}\right) \rho^{m},
$$

and dies off exponentially fast as $m$ goes to infinity. Combined with the first half of the proof, this amounts to

$$
\left|\zeta_{m}-\sum_{|\lambda| \geq \rho} \lambda^{m}\right| \leq m c_{14} \rho^{m}
$$

with $c_{14}$ independent of $m$. Since $\rho<1$ the sum over $m$ uniformly convergent in a small neighbourhood of $f$, and $\sum_{m \in N}\left(\zeta_{m}-\sum_{|\lambda| \geq \rho} \lambda^{m}\right) / m$ converges therefore to an analytic function $\Xi(f)$. However, multiple eigenvalues of $L=L_{f}$ not necessarily depend analytically on $f$ although simple eigenvalues do, but it is well known that the sum of multiple eigenvalues is analytic in $f$. Hence the zeta function which now can be written as

$$
\zeta(f)=e^{\Xi(f)} \prod_{|\lambda| \geq \rho}(1-\lambda(f))^{-1}
$$


has the claimed meromorphic extension to $\left\{f \in C_{u}(\Sigma): P(\mathbb{R} f-u)<0\right\}$ since the finite product on the right hand side is a closed expression.

Since the zeta function and the pressure are invariant under adding a cocycle to $f$, we see using Proposition 1 that the statement of Theorem 4 holds also for two-sided subshifts. Replacing $f$ by $f+\log z$ transforms the pressure of $\mathbb{R} f$ to $P(\mathbb{R} f)+\log |z|$ while the eigenvalues are scaled by a factor $z$.

COROLlaRY 6. $d(z, f)$ is a meromorphic function for $|z|>e^{-P(R f-u)}$ with single poles at $1 / \lambda(f)$ (counting multiplicities), where $\lambda(f)$ are the eigenvalues of $L_{f}$.

A Smale space is a compact metric space with a local product structure and a suitable expansive homeomorphism. This abstract formulation, which generalizes the more familiar Axiom A systems, was given by Ruelle in [11, Ch. 7]. For us the most important fact is that a Smale space $(\Omega, T)$ admits arbitrarily fine Markov partitions. By Smale's spectral decomposition theorem we can always reduce to the case where $T$ or some iterate $T^{k}, k \geq 1$, is topologically mixing on compact subspaces of $\Omega$, see Bowen [2, Theorem 3.5]. Let $(\Omega, T)$ be topologically mixing. Hence there exists a subshift of finite type $(\Sigma, \sigma)$ and a continuous map $\pi: \Sigma \rightarrow \Omega$ such that $T \pi=\pi \sigma$ and where $\pi$ is finite to one and one to one almost everywhere (with respect to ergodic measures of full support). For details see [2]. The construction of the partition is by no means unique. With a suitably chosen metric on $\Sigma$, say for some $\alpha_{0}>0$, the surjection $\pi$ is Lipshitz continuous (cf. [11, p. 130]). A Hölder continuous function $F: \Omega \rightarrow \mathbb{C}$ with Hölder exponent $\beta \in(0,1]$ lifts to some $f=F \pi \in$ $C_{\theta}(\Sigma)$, where the modulus of continuity is the constant $\theta=\beta \alpha_{0}, 0<\theta \leq \alpha_{0}$. With Manning's product formula [6] the results of this section immediately carry over to Axiom A diffeomorphisms in general. For the following corollary compare also [11, pp. 140-142]. Note that in doing the transition from the two-sided to the one-sided problem we lost some regularity in Proposition 1.

Corollary 7. Let $(\Omega, T)$ be a topologically mixing Smale space and $F: M \rightarrow \mathbb{C}$ a complex Hölder continuous function with Hölder exponent $\beta$. Then the zeta function $d(z, F)$ defined as above is analytic and non-zero for $|z|<e^{-P(R F)}$ and has a meromorphic continuation to $|z|<e^{\frac{1}{2} \gamma \beta-P(R F)}$, where $e^{-\gamma}, \gamma>0$, is the rate with which $T, T^{-1}$ contract in the stable and unstable directions on $\Omega$.

\section{Suspended flows}

Let $\theta$ be some positive number. In this section we consider the classical case where the modulus of continuity is a constant $u=\theta \cdot \nabla$. We start off with the two-sided subshift $\Sigma$, a real and strictly positive $r \in C_{\theta}(\Sigma)$ and define on

$$
\mathbf{\Sigma}_{r}=\{(x, t) \in \Sigma \times \mathbb{R}: 0 \leq t \leq r(x)\},
$$

the suspended flow $\varphi_{t}: \Sigma_{r} \rightarrow \Sigma_{r}, t \in \mathbb{R}$, by setting $\varphi_{t}(x, s)=(x, s+t)$ whenever $0 \leq s$, $s+t \leq r(x)$ and extend it to $t \in \mathbb{R}$ by identifying $(x, r(x))$ with $(\sigma x, 0)$ where $\sigma$ is the ordinary shift on $\Sigma$. The function $r$ is often referred to as the ceiling or return function of the suspension $\varphi_{i}$. We give $\boldsymbol{\Sigma}_{r}$ the product topology and consider only suspended flows with more than one closed orbit (weak mixing). 
The pressure of a real valued continuous function $F$ on $\boldsymbol{\Sigma}_{r}$ is defined by the variational principle as

$$
P^{*}(F)=\sup _{\rho}\left(h^{*}(\rho)+\rho(F)\right)
$$

where $\rho$ runs over all $\varphi_{t}$-invariant probability measures on $\boldsymbol{\Sigma}_{r}$. A flow invariant measure $\bar{\mu}$ is of the form $\mu \times \Lambda / \mu(r)$, where $\mu$ is a shift invariant measure on $\Sigma$ and $\Lambda$ is the Lebesgue measure on $\mathbb{R}$. If we denote by $h(\mu)$ the measure theoretic entropy with respect to $\mu$ and by $h^{*}(\bar{\mu})$ the measure theoretic entropy of the time one map $\varphi_{1}$ with respect to $\bar{\mu}$, then we have by a theorem of Abramov [1] the identity $h^{*}(\bar{\mu})=h(\mu) / \mu(r)$, If we put $P_{\bar{\mu}}^{*}(F)$ for $\bar{\mu}(F)+h^{*}(\bar{\mu})$ in the flow case and $P_{\mu}(f)=\mu(f)+h(\mu)$ in the discrete case we get by Abramov's formula $P_{\hat{\mu}}^{*}(F)=$ $P_{\mu}(f) / \mu(r)$, where $f(x)=\int_{0}^{r(x)} F(x, t) d t$. With $\delta$ as the unique number which satisfies $P(f-\delta r)=\theta$ it follows from the variational principle that $P_{\mu}(f-\delta r) \leq \theta$ for all shift invariant probability measures $\mu$ on $\Sigma$, therefore

$$
P_{\bar{\mu}}^{*}(F)-\delta=P_{\bar{\mu}}^{*}(F-\delta)=P_{\mu}(f-\delta r) / \mu(r) \leq \theta / \mu(r)
$$

for all flow invariant measure $\bar{\mu}=\mu \times \Lambda / \mu(r)$, and

$$
\delta=\sup _{\bar{\mu}}\left\{P_{\bar{\mu}}^{*}(F)-\theta / \mu(r)\right\}=\sup _{\mu} P_{\mu}(f-\theta) / \mu(r) .
$$

Let $F$ now be complex and denote by $\ell(\mathscr{O})$ the length of a closed orbit $\mathcal{O}$. The zeta function is then defined by

$$
d^{*}(z, F)=\prod_{O}\left(1-\exp \int_{0}^{\ell(O)}\left(F\left(\varphi, x_{O}\right)-z\right) d t\right\}^{-1}
$$

for $x_{\mathscr{O}} \in \mathcal{O}$ and $z$ a complex variable. The product is over all closed orbits and converges to an analytic and non-zero function whenever $\mathbb{R} z>P^{*}(\mathbb{R} F)$. If $F$ is a nice enough function such that $f$ is in $C_{\theta}(\Sigma)$, we have $d^{*}(z, F)=\zeta(f-z r)$. For $\delta<P(\mathbb{R} f)$ the unique number which satisfies $P(\mathbb{R} f-\delta r)=\theta$ we get by Theorem 4 that $d^{*}(z, F)$ has a meromorphic continuation to the complex halfplane $\mathbb{R} z>\delta$ with poles whenever $L_{f+z r}$ has 1 as eigenvalue.

Proposition 8. $d^{*}(z, F)$ is analytic and non-zero for $\mathbb{R} z>P^{*}(\mathbb{R} F)$ and has a meromorphic extension to the halfplane $\{z \in \mathbb{C}: \mathbb{R} z>\delta\}$ with poles whenever $L_{f+z r}$ has 1 as eigenvalue, where $\delta=\sup _{\mu}\{(\mu(\mathbb{R} f-\theta)+h(\mu)) / \mu(r)\}$ and the supremum is over shift invariant probability measures on $\Sigma$.

\section{Axiom A flows}

We apply the result of $\S \S 3$ and 4 to Axiom A flows. Let $M$ be a Riemannian manifold and let $\Phi_{t}: M \rightarrow M, t \in \mathbb{R}$, be a smooth flow. A compact invariant subset $\Delta \subset M$ that contains no fixed points is called hyperbolic if the tangent bundle restricted to $\Delta$ is a Whitney sum

$$
T_{\Delta} M=E+E_{s}+E_{u},
$$

of three $D \Phi$, invariant sub-bundles, where $E$ is the one dimensional bundle tangent 
to the flow and $E_{s}, E_{u}$ are contracting and expanding respectively, i.e.

$$
\begin{aligned}
\left\|D \Phi_{t}(v)\right\| \leq C e^{-\gamma t}\|v\| & \text { for } v \in E_{s}, t \geq 0, \\
\left\|D \Phi_{-t}(v)\right\| \leq C e^{-\gamma t}\|v\| & \text { for } v \in E_{u}, t \geq 0,
\end{aligned}
$$

where $C$ is a constant and $\gamma>0$ is a contracting parameter of the flow. The hyperbolic set $\Delta$ is called basic if periodic orbits are dense, $\Phi_{t}$ restricted to $\Delta$ is topologically transitive and $\Delta=\bigcap_{t \in R} \Phi_{t} U$ for an open neighbourhood $U$ of $\Delta$. We say $\Delta$ is non-trivial if it contains more than one closed orbit.

For Hölder continuous $F: \Delta \rightarrow \mathbb{C}$ with Hölder $\beta \in(0,1]$ we define as in the last section a zeta function $Z(z)$ by integrating along closed orbits. By introducing transverse Markovian sections $H_{i}$ on $M$ and tracing them under the forward flow $\Phi_{t}, t>0$, one derives a suspended flow $\varphi_{t}: \Sigma_{r} \rightarrow \Sigma_{r}$ which via $\pi: \Sigma_{r} \rightarrow \Delta$ is semiconjugate to $\Phi_{t}$, that is $\pi \varphi_{t}=\Phi_{t} \pi, t \in \mathbb{R}$. The ceiling function $r$ measures the 'time' it takes for points of one section $H_{i}$ to flow up to the next. Moreover, if $\Phi_{t}$ is weak mixing so is $\varphi_{1}$. A detailed account of the construction is given in Bowen [3] from where we need the following Lemma 1.5.

LEMMA 9. There exists a constant $c_{0}$ so that for $\varepsilon>0$ there exists $\varepsilon^{\prime}>0$, such that if for $\xi, \zeta \in \Delta$ there exists a continuous $s: \mathbb{R} \rightarrow \mathbb{R}$ with $s(0)=0$ and $d\left(\Phi_{t} \xi, \Phi_{s(t)} \zeta\right) \leq \varepsilon^{\prime}$, $|t| \leq L, L>0$ then

$$
d\left(\xi, \Phi_{v} \zeta\right) \leq c_{0} e^{-\gamma L}
$$

for some $|v| \leq \varepsilon$.

We apply this result to our situation using that $\Phi_{t}$ is semiconjugate to the suspension $\varphi_{t}$. As a result we are able to determine the modulus of continuity of the ceiling function of $\varphi_{t}$. The map $\pi$ from the shift $\Sigma$ to $\bigcup_{i} H_{i}$ is Hölder continuous. Let $x, y \in \Sigma$ and $\xi, \zeta \in \Delta$ such that $\pi x=\xi, \pi y=\zeta$. If $x_{i}=y_{i}$ for $|i| \leq n, n \geq 1$, then $d\left(\Phi_{t} \xi, \Phi_{s(t)} \zeta\right) \leq \varepsilon^{\prime}$, where we choose $\varepsilon^{\prime}$ to be the size of the cross-sections $H_{i}$ which can be made arbitrarily small, and $t$ satisfies $-r^{-n}(x) \leq t \leq r^{n}(x)$, with $r^{-n}=$ $r \sigma^{-1}+\cdots+r \sigma^{-n}$. The function $s$ is determined such that $s\left(r^{k}(x)\right)=r^{k}(y), k=$ $-n, \ldots, n$, and made continuous and monotone in between. Hence by Lemma 9 $d(\xi, \zeta) \leq c_{0} e^{-\gamma L}, L=\min \left(r^{-n}(x), r^{n}(x)\right)$, and since the ceiling function is Lipshitz continuous as a function on the cross-sections $H_{i}$, it follows that

$$
\operatorname{var}_{n} r(x)=\sup \left\{|r(x)-r(z)|: z \in \Sigma, z_{i}=x_{i} \forall|i| \leq n\right\} \leq c_{1} d(\xi, \zeta) \leq c_{2} e^{-\gamma L},
$$

amd consequently $r$ has modulus of continuity $\frac{1}{2} \gamma r$. By the same argument one shows that the lifted function $f(x)=\int_{0}^{r(x)} F \circ \pi(x, t) d t$ has modulus of continuity $\frac{1}{2} \gamma \beta r$. We now use Proposition 1 to reduce the problem to one on one-sided shift spaces and conclude by Theorem 4 that the zeta function of the suspended flow can be meromorphically extended to complex $z$ for which $P\left(\mathbb{R}(f-z r)-\frac{1}{2} \gamma \beta r\right)=$ $P(\mathbb{R} F)-\mathbb{R} z-\frac{1}{2} \gamma \beta<0$, where $P(\mathbb{R} F)$ is the pressure of $\mathbb{R} F$ for the flow $\Phi$, as defined by the variational principle. Unfortunately $\pi: \Sigma_{r} \rightarrow \Delta$ does not necessarily preserve the least period of a closed orbit. However, Bowen, who carried further Manning's construction of subshifts for diffeomorphisms, saved the situation by constructing a similar collection of suspensions. 
Proposition 10. (Bowen-Manning.) There exist finitely many suspended flows $\varphi_{i}^{(i)}: \Sigma_{(i)} \rightarrow \Sigma_{(i)}, i=0, \ldots, k, \varphi_{l}^{(0)}=\varphi_{t}$, and projections $\pi_{(i)}: \Sigma_{(i)} \rightarrow \Delta$, such that $\varphi_{i}^{(i)} \pi_{(i)}=$ $\pi_{(i)} \Phi_{t}, t \in \mathbb{R}$, and

$$
Z(z)=Z_{0}(z) \prod_{i \geq 1} Z_{i}(z)^{\varepsilon(i)}
$$

where $Z_{i}(z)$ is the zeta function for the suspension $\varphi_{t}^{(i)}$ and where $\varepsilon(i)= \pm 1, i=1, \ldots, k$. Moreover, the suspensions $\varphi_{t}^{(i)}$ have topological entropy strictly less than $h\left(\Phi_{t}\right)=h^{*}\left(\varphi_{t}\right)$.

THEOREM 11. Let $\Phi_{t}$ be a $C^{1}$ Axiom A flow with non-trivial basic set $\Delta$ and contraction parameter $\gamma$ and $F: \Delta \rightarrow \mathbb{C}$ a Hölder continuous function with Hölder exponent $\beta \in(0,1]$. Then the zeta function $Z(z)$ is non-zero and analytic for $\mathbb{R} z>P(\mathbb{R} F)$ and has $a$ meromorphic extension to the halfplane $\left\{z \in \mathbb{C}: \mathbb{R} z>P(\mathbb{R} F)-\frac{1}{2} \gamma \beta\right\}$.

Acknowledgement. This paper was written during a stay at the IHES. I acknowledge the hospitality and the financial support which I was granted. I would also like to thank D. Ruelle and P. Walters for conversations.

\section{REFERENCES}

[1] L. M. Abramov. On the entropy of a flow. Dokl. Akad. Nauk. SSSR 128 (1959), 873-876. Amer. Math. Soc. Transl. 249 (1966), 167-170.

[2] R. Bowen. Equilibrium States and the Ergodic Theory of Anosov Diffeomorphisms. S.L.N. No. 470. Springer, New York, 1975.

[3] R. Bowen. Sybolic dynamics for hyperbolic flows. Amer. J. Math. 95 (1973), 429-459.

[4] G. Gallovotti. Funzione zeta ed insiemi basilar; Accad. Linei. Rend. Sc. fisimat. e nat. 61 (1976), 309-317.

[5] F. Hofbauer \& G. Keller. Zeta functions and transfer operators for piecewise linear transformations; J. reine angew. Math. 352 (1984), 100-113.

[6] A. Manning. Axiom A diffeomorphisms have rational zeta functions. Bull. Lond. Math. Soc. 3 (1971), 215-220.

[7] R. D. Nussbaum. The radius of the essential spectrum. Duke Math. J. 37 (1970), 473-478.

[8] W. Parry. An analogue of the prime number theorem for shifts of finite type and their suspension. Israel J. Math. 45 (1983), 41-52.

[9] W. Parry. Bowen's equidistribution theory and the Dirichlet density theorem. Ergod. Th. \& Dynam. Syst. 4 (1984), 117-134.

[10] M. Pollicott. Meromorphic extensions of generalised zeta functions. Invent. Math. 85 (1986), 147-164.

[11] D. Ruelle. Thermodynamic Formalism. Addison-Wesley, Reading, 1978.

[12] D. Ruelle. One dimensional Gibbs' states and Axiom A diffeomorphisms. J. Diff. Geom. 25 (1987), 117-137.

[13] Ya. G. Sinai. Gibbs' measures in ergodic theory. Russ. Math. Surv. 27(4) (1972), 21-69.

[14] P. Walters. An Introduction to Ergodic Theory. G.T.M. No. 79. Springer, New York, 1981. 